\title{
Tonal frequency shifts and gaps in acoustic stimulation as reflex-modifying events
}

\author{
JACQUELYN CRANNEY, HOWARD S. HOFFMAN, and MICHELLE E. COHEN \\ Bryn Mawr College, Bryn Mawr, Pennsylvania
}

When a relatively weak signal, such as a mild tone, precedes an intense reflex-eliciting stimulus by an appropriate interval (about $100 \mathrm{msec}$ ), the amplitude of the elicited reaction is often reduced. It was found that in student volunteers a brief gap in a steady pure tone that occurred 150 msec prior to a mild tap to the glabella (the flat region between the eyebrows) could inhibit the eyeblink elicited by the tap. It was also found that a shift in tonal frequency across a gap in a tone was more inhibitory than a gap with no frequency shift, but it was no more inhibitory than the onset of the short second tone alone. The final study determined the minimum amount of frequency shift required to produce an additional inhibitory effect above that of a gap alone. The findings are discussed in terms of various aspects of sensory processing.

When a relatively weak signal, such as a mild tone, precedes an intense reflex-eliciting stimulus by an appropriate interval (about $100 \mathrm{msec}$ ), the amplitude of the elicited reaction is often reduced. This reflexmodification effect is characteristic of a wide variety of birds and mammals, including man. It does not depend upon learning. It is seen the first time that the reflex-modifying event occurs. Moreover, the effect does not require the active cooperation of the subject. In humans, reflex modification has been assessed while subjects were sleeping (Silverstein \& Graham, 1979), while they were reading (Dykman \& Ison, 1979), and (as with the present research) while they were watching a sequence of color slides (Hoffman, Cohen, \& Stitt, 1981). All of these factors point to the efficacy of reflex modification as a tool in the objective analysis of sensory processing.

In the research reported here, we have sought to examine the reflex modification afforded by relatively complex patterned acoustic events. Previous investigations of reflex modification have mainly employed unidimensional, steady state pure tones or noise bands as reflex-modifying events, and the details of their effects have been extensively studied (see Hoffman \& Ison, 1980, for a review). However, the effects of patterned or multidimensional reflexmodifying signals have received little attention, despite the relevance that such information might have to an understanding of the behavioral effects of naturally occurring patterned sounds, such a speech.

Among the few previous studies on the reflexmodifying effects of complex changes in acoustic sig-

This research was supported by Grant HD 10511 from the National Institutes of Health, directed by Howard S. Hoffman. The bulk of this research was conducted by the first author as part of her doctoral dissertation at Bryn Mawr College. Requests for reprints should be sent to Howard S. Hoffman, Department of Psychology, Bryn Mawr College, Bryn Mawr, PA 19010. nals is that by Stitt, Hoffman, Marsh, \& Boskoff (1974). These investigators reported a reduction in startle amplitude in rats following instantaneous shifts between low-band and high-band random noise just prior to a startle-eliciting stimulus.

More recently Ison (1982) reported that a brief (4msec) gap in an otherwise continuous noise could also inhibit a subsequently elicited startle reaction. Moreover, Kellogg, Ison, and Miller (1983) reported that prenatal diazepam exposure in rats retarded the development of auditory temporal resolution, as indexed by the effectiveness of acoustic gaps as startle inhibitors. It is of special interest that Ison and Pinckney (1983) reported that the minimum gap required to reliably produce inhibition of an elicited eyeblink in humans (again approximately $4 \mathrm{msec}$ ) corresponds to the values reported in various assessments of the threshold for gap detection, as measured with standard psychophysical procedures (Penner, 1975; Perrott \& Williams, 1971; Plomp, 1964; Smiarowski \& Carhart, 1976; Williams, Elfner, \& Howse, 1979).

The current series of experiments was designed to extend these lines of investigation by using gaps and shifts in the frequency of tones (rather than noise) as the reflex-modifying event. More specifically, the work sought to document the reflex modification engendered when student volunteers were exposed to various patterned changes in an ongoing pure tone, just prior to the presentation of an eyeblink-eliciting tap to the glabella (the flattened area of skin between the eyebrows).

The advantage to be gained by using tones (rather than noise) was twofold. First, it would make it possible to better specify the exact acoustic parameters of the reflex-modifying events as they varied over time. Second, it would facilitate the interpretation of the data. Pure tones ordinarily have more clearly de- 
fined and better understood neurophysiological consequences than do the broadband signals used in most prior studies.

\section{EXPERIMENT 1}

The aim of the first study was to determine if the inhibitory effect of a gap in an otherwise ongoing noise band (Ison \& Pinckney, 1983) could also be found using pure tones.

\section{Method}

Subjects. Twelve graduate and undergraduate students from Bryn Mawr College ( 11 females and 1 male) participated in this experiment as paid volunteers.

Apparatus. All research was conducted in an IAC double-walled sound-treated room with an ambient noise level below $25 \mathrm{dBA}$. This room was suitably treated and lighted. It was also equipped with a rear-projection screen so that $35-\mathrm{mm}$ slides could be projected from the control area outside the room. A closed-circuit television camera and an intercom permitted continuous monitoring of the subject.

Acoustic stimuli employed in this study were generated, shaped, amplified, and timed by Coulbourn programming modules. They were delivered to the subject through THD-39 earphones fitted with MX-41/AR cushions. The earphones and test stimuli were calibrated with a General Radio precision sound-level meter (Model 1561-A) fitted with a P-7 microphone and ANSI type coupler. All intensities reported here are sound-pressure level (SPL re $20 \mathrm{~N} / \mathrm{m}^{2}$ ).

Controlled taps to the subject's glabella were delivered by a device described in detail elsewhere (Marsh, Hoffman, \& Stitt, 1979). Briefly, this unit consisted of a miniature solenoid with a small ( $5 \mathrm{~cm}$ in diameter) ball of silicone rubber securely fastened to its plunger. When the solenoid was activated, the ball was thrust forward with an impulse (in newtons/sec) that, as measured with a ballistic pendulum, was equal to .95 times the voltage applied.

Eyeblinks were measured by a device that has also been described elsewhere (Marsh et al., 1979). Briefly, the monitor consisted of a miniature optical unit $(1 \times .5 \times .5 \mathrm{~cm})$ that contained an infrared light-emitting diode and a sensitive phototransistor pickup. The peak output of the eyeblink-monitoring device during the 150 -msec period that began with the onset of the 50 -msec tap was read by a peak detector (Coulbourn Module S76-31) and then digitized and strobed to a TRS80 Radio Shack microcomputer. The 150-msec period prior to onset of the tap was also monitored, and if a voluntary blink was detected, as defined by a criterion response of at least $1 \mathrm{~V}$, that trial was immediately repeated. Trial presentation order was always in randomized blocks, and each trial was initiated by the microcomputer.

The tap and eyeblink monitoring devices were firmly attached to a lightweight headband. The headband was adjusted on the subject so that the silicone ball on the solenoid was approximately $3 \mathrm{~mm}$ away from the glabella and the eyeblink monitoring device was directly in front of the subject's left eye (approximately $5 \mathrm{~mm}$ away from the ends of the subject's eyelashes).

Stimulus conditions. In this and in most of the subsequent experiments, the baseline condition was a tap to the glabella (produced by delivering a 16-V dc, 50-msec pulse to the tap solenoid) in the absence of prior or concurrent acoustic stimulation. Responses to this tap were compared with responses to the same tap when the tap was either accompanied or preceded by a given acoustic stimulus. Some major methodological concerns in this work were that the acoustic stimuli be artifact free, that their parameters be within dimensional ranges relevant to speech, and that their parameters also be consistent with the documented technology of reflex modification. Tone frequencies were 1.0 and $2.5 \mathrm{kHz}$. These frequencies are within the range of normal speech yet are easily dis- criminable from each other. It was also arranged that all tone onsets and offsets would be moderately slow (e.g., linear rise/fall time equaled $10 \mathrm{msec}$ ). This avoided the acoustic transients (clicklike sounds) that occur whenever a pure tone undergoes a rapid change in intensity and/or frequency (see Licklider, 1951, for detailed discussion of this problem).

The several stimulus conditions are presented schematically in Figure 1. In experiment 1, two basic acoustic stimulus conditions were employed: (1) constant tone and (2) gap. The constant tone condition was a 10-sec tone ending with an eyeblink-eliciting tap. In this stimulus condition, the tone offset (the beginning of the 10msec fall of the 10-sec tone) coincided with the onset of a $50-\mathrm{msec}$ tap. The gap condition also commenced with a 10 -sec tone but was followed by a number of critical events: a $10-\mathrm{msec}$ fall in the intensity of the first tone followed by $4 \mathrm{msec}$ of silence (the gap) and then the 10-msec onset of a $150-\mathrm{msec}$ second tone. The full offset of the second tone required $10 \mathrm{msec}$, and it began with the onset of the 50-msec tap. In Experiment 1 (in contrast to the following studies), the second tone was always the same frequency as the first tone. All tones were $70 \mathrm{~dB}$ in intensity.

Overall, Experiment 1 contained five stimulus conditions: (1) startle-eliciting stimulus only-a tap to the glabella with no prior or concurrent acoustic stimulation (tap-alone condition); (2) a 10-sec steady $1.0-\mathrm{kHz}$ tone that offset simultaneously with the onset of the tap (constant-tone condition, $1.0 \mathrm{kHz}$ ); (3) a 10 sec $1.0-\mathrm{kHz}$ tone followed by a brief $(4-\mathrm{msec})$ silent period and then the onset of a second $150-\mathrm{msec} 1.0-\mathrm{kHz}$ tone that offset simultaneously with the presentation of the tap (gap condition, $1.0 \mathrm{kHz}$; (4) a $10-\mathrm{sec}$ steady $2.5-\mathrm{kHz}$ tone that offset simultaneously with the onset of the tap (constant tone condition, $2.5 \mathrm{kHz}$ ); (5) a $10-\mathrm{sec} 2.5-\mathrm{kHz}$ tone followed by a brief ( $4 \mathrm{msec})$ silent period and then the onset of a second $150-\mathrm{msec}$ tone that

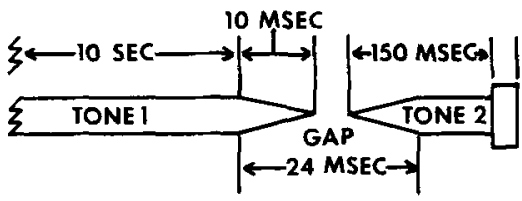

TAP ALONE

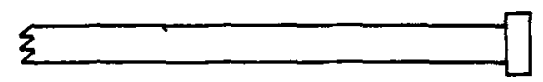

CONSTANT TONE
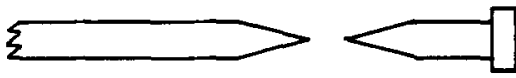

GAP (NO SHIFT)
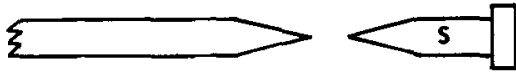

SHIFT (+ GAP)

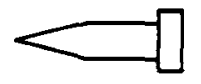

ONSET

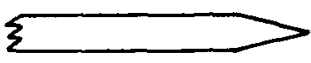

OFFSET

Figure 1. The general set of conditions used in this series of experiments (not to scale). "S" signifies a shift in frequency. 
offset simultaneously with the onset of the tap (gap condition, $2.5 \mathrm{kHz}$ ). With this arrangement, Conditions 4 and 5 duplicated Conditions 2 and 3 except for the difference in tone frequency.

Procedure. After each subject had been apprised of the nature of the research and had agreed to participate, he or she was seated in the IAC room and fitted with earphones and the headband holding the tap and eyeblink monitoring devices. The subjects received 10 binaural presentations of each of the five stimulus conditions in a random order. Trials occurred at intervals that varied between 15 and $25 \mathrm{sec}$, with an average time of $20 \mathrm{sec}$. The trials were arranged so that each kind of stimulus condition appeared once in each block of five trials in an order that varied from trial block to trial block. The subjects were told that they would receive various tones and taps while they were watching a sequence of color slides on a variety of topics. They were told that their blinks to the taps were being measured but that they need not be concerned about them, and that they should relax and enjoy the slides. All tones and taps were presented in the intervals between slide changes.

\section{Results and Discussion}

The basic datum unit in these experiments was the mean amplitude of eyeblink (in volts) across trials for each condition for each subject. Figure 2 presents the mear' response amplitude, averaged across the 12 subjects for each of the five stimulus conditions. The 'two constant tone conditions produced eyeblinks of slightly greater amplitude than did the tap-alone condition, whereas the two gap conditions produced eyeblinks of slightly smaller amplitude than did the tap-alone condition. A one-way repeated measures analysis of variance confirmed that there were statistically reliable differences among the conditions $[F(4,44)=7.48$, $p<.05]$. A subsequent NewmanKeuls analysis $(p<.05)$ indicated that responding in the two gap conditions was reliably lower than responding in the other three conditions (constant-tone conditions and tap-alone condition). That is, the two gap conditions produced significant inhibition of the

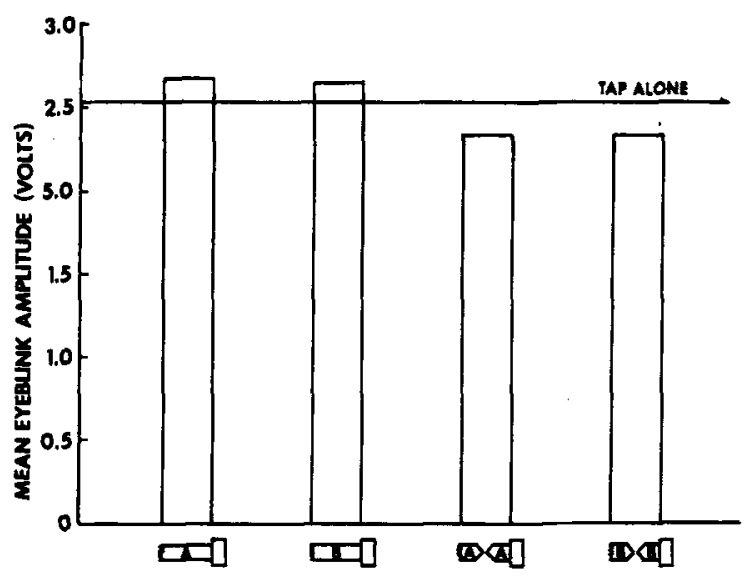

Figure 2. Mean amplitude of tap-elleited eyeblinks (across subjects) for each of the several stimulus configurations lllustrated schematically below the horizontal axis of the figure. $(A=1.0$ kHz tone, $B=2.5-k H z$ tone. The vertically oriented rectangles = the glabella tap. See Figure 1 for a complete deacription. The horzontal line in the figure = the mean amplitude of eyeblinks ellelted by the tap without prior or concurrent acoustic stimulation.) elicited eyeblinks. However, the observed differences between the two constant-tone conditions and the tap-alone condition were not statistically reliable, providing no evidence of facilitation (Hoffman \& Searle, 1965) of the eyeblink response by the presence of the constant tone. To date, facilitation has been reported only with rats (e.g., Hoffman \& Wible, 1969), so the current failure to observe facilitation in humans is not surprising. There were no significant differences between the two gap conditions, or between the two constant-tone conditions, implying that there were no differential effects of tone frequency in this experiment. In general, these results extend those of Ison and Pinckney (1983) by revealing that the gap inhibitory effect can be obtained using pure tones.

\section{EXPERIMENT 2}

The next step in this series of studies was to determine whether a shift in tonal frequency across a gap in acoustic stimulation would lead to any further reflex inhibition than a gap condition with no frequency shift. Previous work (Marsh, Hoffman, Stitt, \& Schwartz, 1975; Stitt et al., 1974) had already indicated that shifts between high- and low-frequency bands of noise could inhibit a startle reflex; the question addressed here was whether similar effects could be obtained when the basic acoustic signal was a tone rather than noise, and when the frequency shift occurred across a gap in a signal that had a slow enough rise time $(20 \mathrm{msec})$ that it would be transient free. Given that the effects of stimulus change often appear to be additive in terms of amount of startle amplitude reduction (Ison, Zuckerman, \& Russo, 1975), it was hypothesized that the frequency shift condition would lead to greater inhibition than would the gap/no-shift condition.

\section{Method}

Subjects and Apparntus. Five female graduate and undergraduate subjects from Bryn Mawr College participated in this experiment as paid volunteers. The apparatus was similar to that used in Experiment 1, with the exception that the trials were initiated and the responses recorded manually (see, e.g., Cohen, Hoffman, \& Stitt, 1981) rather than by computer.

Stimulus conditions. In this experiment, each subject was presented with two basic stimulus configurations, as outlined in Figure 1: (1) a 10-sec tone in which a 4-msec silent period (gap) occurred 154 msec prior to the glabella tap (gap/no-shift condition); (2) a 10-sec tone in which a 4-msec gap occurred $154 \mathrm{msec}$ prior to a tap, with a shift in tonal frequency coincident with the gap (frequency shift condition). As in the previous study, the lowfrequency tone was $1.0 \mathrm{kHz}$ and the high-frequency tone was $2.5 \mathrm{kHz}$. All tones were $60 \mathrm{~dB}$ with a 20 -msec rise/fall time. Thus, there were four conditions: (1) $2.5-\mathrm{kHz}$ tone with gap followed by a glabella tap, (2) 1.0-kHz tone with gap followed by a tap, (3) tone with gap coincident with a 1.0- to $2.5-\mathrm{kHz}$ shift followed by a tap, and (4) tone with gap coincident with a $2.5-$ to $1.0-\mathrm{kHz}$ shift followed by tap.

Procedure. Each subject received 12 blocks of trials, each block containing a different randomization of the four stimulus condi- 
tions. As in Experiment 1, the subject was permitted to view colored slides throughout the procedure and the ITI was variable (mean $=20 \mathrm{sec}$; range $=15$ to $25 \mathrm{sec}$ ).

\section{Results}

Figure 3 shows the mean amplitude (averaged across subjects) of elicited eyeblinks in each of the four stimulus conditions. It can be seen in this figure that the conditions with the frequency shift produced smaller reflexive eyeblinks than did the two conditions that did not have a frequency shift. A one-way repeated measures analysis of variance confirmed that the differences among the conditions were statistically reliable $[F(3,12)=5.83, p<.05]$. The Newman-Keuls analysis $(p<.05)$ confirmed that the two shift conditions produced significantly lower reflexive eyeblinks than did the two gap/no-shift conditions. As in Experiment 1, neither tone frequency itself nor direction of frequency shift had a different effect. The average voltage levels obtained in this experiment were different from those of the other experiments because of the different apparatus and amplifying devices used.

\section{Discussion}

These results imply that a frequency shift brings additional and perhaps independent reflex-inhibitory processes to the gap condition. It is conceivable that this frequency-shift advantage in inhibition is mediated by a change in the discharge rate of central auditory units, which are known to respond to shifts in frequency (i.e., frequency shift detectors; Nelson, Erulkar, \& Byron, 1966; Whitfield \& Evans, 1965). A test of this notion would compare reflex inhibition across two conditions, one with the standard frequency shift across a gap (shift-gap), the other with the short second tone (onset) but no first tone. As the onset condition does not involve a specific shift in frequency, and hence does not stimulate frequencyshift detectors, one might expect that it would pro-

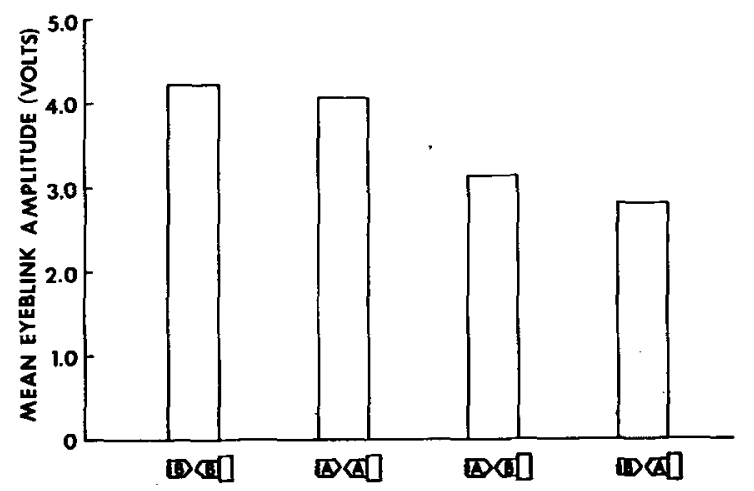

Figure 3. Mean amplitude of tap-elicited eyeblink (across subjects) for each of the several stimulus configurations illustrated schematically below the horizontal axis of the figure. $(A=1.0$ $\mathrm{kHz}$ tone, $\mathrm{B}=2.5-\mathrm{kHz}$ tone. The vertically oriented rectangles = the glabella tap. See figure 1 for a complete description.)

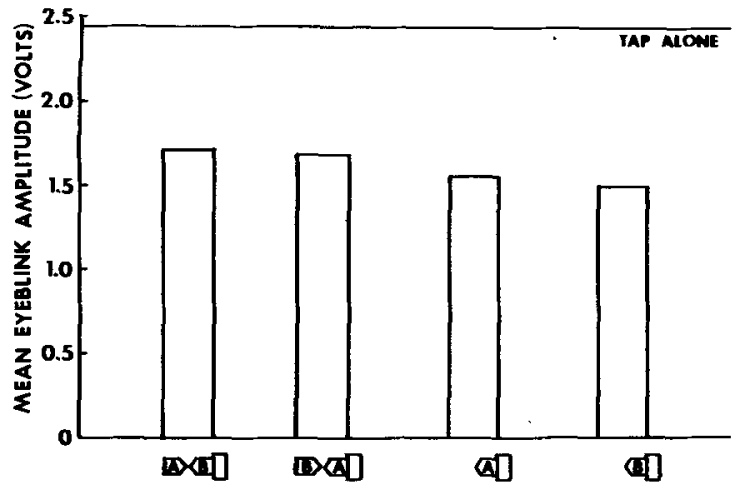

Figure 4. Mean amplitude of tap-elicited eyeblinks (across subjects) for each of the several stimulus conditions illustrated schematically below the horizontal axis of the figure. $(A=1.0-\mathrm{kHz}$ tone, $B=2.5-\mathrm{kHz}$ tone. The vertically oriented rectangles $=$ the glabella tap. See Figure 1 for a complete description. The horizontal line in the figure $=$ the mean amplitude of eyeblinks elicited by the tap without prior or concurrent acoustic stimulation.)

duce less inhibition than the frequency-shift condition. This notion was directly tested in Experiment 3.

\section{EXPERIMENT 3}

\section{Method}

Subjects and Apparatus. Twelve graduate and undergraduate female students from Bryn Mawr College participated in this experiment as paid volunteers. The apparatus was identical to that used in Experiment 1.

Stimulus conditions. During the experimental session, each subject was presented with five basic stimulus configurations (see Figure 1): (1) tap alone, (2) a brief $(150-\mathrm{msec}) 1.0-\mathrm{kHz}$ tone that terminated simultaneously with the onset of the tap $(1.0-\mathrm{kHz}$ onset condition), (3) a brief $(150-\mathrm{msec}) 2.5-\mathrm{kHz}$ tone that terminated simultaneously with the onset of the tap (2.5- $\mathrm{kHz}$ onset condition), (4) a 10-sec tone in which a brief (4-msec) silent period (gap) coincided with an upward frequency shift $(1.0$ to $2.5 \mathrm{kHz})$ in the tone $150 \mathrm{msec}$ prior to the tap (upward shift condition), and ( 5 ) a 10 -sec tone in which a brief (4-msec) gap coincided with a downward frequency shift $(2.5$ to $1.0 \mathrm{kHz})$ in the tone $150 \mathrm{msec}$ prior to the tap (downward shift condition). The intensity of the tones was $70 \mathrm{~dB}$, and the rise/fall time was $10 \mathrm{msec}$.

Procedure. Each subject received 10 blocks of trials, each block consisting of a different random order of the five stimulus conditions. Again, colored slides were shown, and the ITI was variable (mean $=20 \mathrm{sec}$; range $=15$ to $25 \mathrm{sec}$ ).

\section{Results}

Figure 4 shows the mean amplitude of eyeblink (in volts) averaged across subjects for each of the five stimulus conditions. Relative to the tap-alone condition, all prestimulation conditions led to substantial inhibition. A one-way repeated measures analysis of variance confirmed that overall the differences among the conditions were statistically reliable $[F(4,44)=19.25, p<.05]$. Subsequent NewmanKeuls analysis of the means $(p<.05)$ revealed that the tap-alone condition produced significantly greater startle response than did any of the other conditions, but there were no significant differences among the remaining conditions. As in Experiments 
1 and 2 , frequency of the tone did not have a differential effect.

\section{Discussion}

The major finding of interest in this experiment is that the frequency-shift condition produced no greater inhibition than did the onset condition. This finding provides no support for the original hypothesis, that inhibition by a shift in frequency entails the activation of frequency-shift detectors. While there is considerable evidence for the existence of shift detectors, it has been found that the majority of the frequency-shift detectors do not respond exclusively to frequency shifts; they also respond to onsets of tones (Nelson et al., 1966; Whitfield \& Evans, 1965). In such circumstances, one might not necessarily expect more inhibition by a frequency shift than occurs with a tone onset. Another factor to be considered is the neuroanatomical location of these shift-detector units, and the role such structures might play in reflex modification. As indicated in the physiological literature (e.g., Nelson et al., 1966; Whitfield \& Evans, 1965), the greater proportion of frequency shift units are found in auditory nuclei above the meso-diencephalic junction. This seems important because there is evidence that the integrity of these structures is not necessary for reflex inhibition to occur [e.g., reflex inhibition survives knife cuts at the level of the meso-diencephalic junction (Davis \& Gendelman, 1977) and lesioning of telencephalic nuclei and cortex (Groves, Boyle, Welker, \& Miller, 1974; Kemble \& Ison, 1971)]. Since the data obtained here provided no suggestion that shift detectors are contributing to reflex modification, they are consistent with these considerations.

\section{EXPERIMENT 4}

The findings in the first three experiments were that a gap in a tone is inhibitory, that a frequency shift across the gap is more inhibitory than no frequency shift across a gap, and that an onset is just as inhibitory as a frequency shift across a gap. These findings lead to the inference that the onset condition will result in greater inhibition than will the gap/noshift condition. This inference, however, is based on a comparison of the results of separate studies involving different groups of subjects. Stronger evidence for this inference would be provided if it could be demonstrated that in the same subjects the onset condition produced the same amount of inhibition as the shift-gap condition but greater inhibition than the gap/no-shift condition. Given that a tone onset produced as much inhibition as a frequency shift across a gap, it was conceivable that a tone of fset might also produce an equivalent amount of inhibition. Thus, the current experiment incorporated all of the conditions of interest: gap, shift, onset, offset, constant, and tap alone. Finally, by combining all of these conditions, Experiment 4 provided an opportunity to replicate the procedures that had been used in Experiments 1,2 , and 3.

\section{Method}

Stimulus conditions. In Experiment 4 each subject was presented with six basic stimulus conditions, as depicted in Figure 1: ticipated in this experiment as paid volunteers. The apparatus was the same as that used in Experiment 1.

Stimulus Conditions. In Experiment 4 each subject was presented with six basic stimulus conditions, as depicted in Figure 1: (1) tap alone, (2) a 10-sec steady tone ending with the tap (constant tone condition), (3) a 10-sec tone with a brief (4-msec) silent period (gap) $154 \mathrm{msec}$ prior to the tap (gap condition), (4) a 10-sec tone in which a brief (4-msec) gap coincided with a frequency shift in the tone $150 \mathrm{msec}$ prior to the tap (shift condition), (5) a brief $(150$-msec) tone that offset simultaneously with the onset of the tap (onset condition), and (6) a 10-sec tone which offset $154 \mathrm{msec}$ prior to the tap (offset condition). As in the previous studies, all stimulus configurations involving a tone were counterbalanced for frequency ( 1.0 and $2.5 \mathrm{kHz}$ ), making a total of 11 stimulus conditions. In all configurations, tone intensity was $70 \mathrm{~dB}$, and the rise/fall time was $10 \mathrm{msec}$.

Procedure. Subjects participated in two sessions; in each session, four blocks of trials, each block consisting of a different random order of the 11 stimulus conditions, were presented. Again, colored slides were shown and the ITI was variable (mean $=20 \mathrm{sec}$; range $=15$ to $25 \mathrm{sec}$ ).

\section{Results}

Figure 5 presents the mean eyeblink amplitude for the six basic stimulus conditions, averaged across subjects, session, and tone frequency. Although the gap, onset, offset, and shift conditions each have an inhibitory effect, the inhibitory effect of the shift and onset conditions is greater than that of the gap and offset conditions. A two-way (condition $x$ session) repeated measures analysis of variance of eyeblink responses revealed that there were no main effects

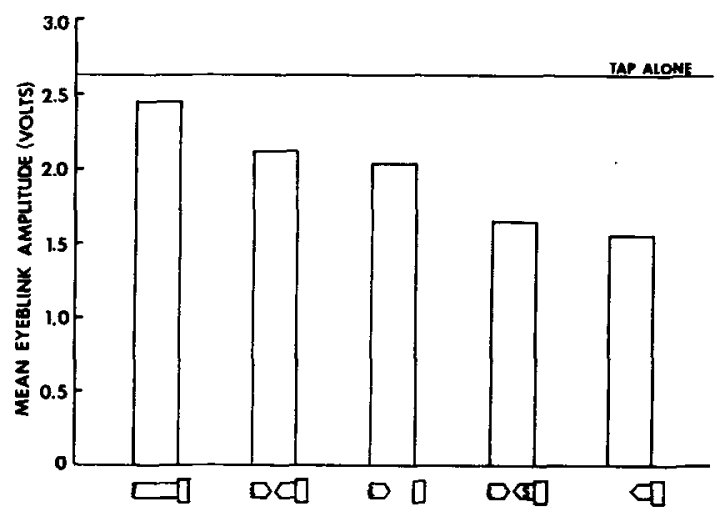

Figure 5. Mean amplitude of tap-elicited eyeblinks (across subjeets) for each of the several stimulus configurations lllustrated schematically below the horizontal axis of the figure. (The vertically oriented rectangles = the tap; the horkontally oriented rectangles $=$ the tone. $S=$ shift in frequency of the second tone in the shift-gap condition. See Figure 1 for a complete description. The horizontal line in the figure $=$ mean amplitude of eyeblinks elicited by the tap without prior or concurrent acoustic stimulation.) 
or interactions involving the session factor. Moreover, a Newman-Keuls analysis $(\mathrm{p}<.05)$ of the mean responses in each of the conditions (averaged across sessions) showed no differential effect of frequency. Given that there were no effects of session or tone frequency, a one-way repeated measures analysis of variance of the mean responses for the six basic stimulus conditions, collapsed across frequency and session, was conducted. This analysis confirmed that there were significant differences between the basic stimulus conditions $[F(5,55)=18.31, p<.05]$. A subsequent Newman-Keuls analysis $(p<.05)$ revealed the following effects: (1) Eyeblink amplitudes in the tap-alone and constant-tone conditions were not significantly different, and were greater than eyeblink amplitudes in all other conditions; (2) eyeblink amplitude in the gap and offset conditions were equivalent; and (3) eyeblink amplitudes in the shift and onset conditions were equivalent, and less than that in all other conditions.

\section{Discussion}

These findings replicate those of the first three experiments, and extend them in a number of ways. As expected, the onset condition produced significantly greater inhibition than the gap condition. The amount of inhibition produced by the offset condition was equal to that produced by the gap-no shift condition and less than that produced by the shiftgap and onset conditions. The finding that the onset condition produced more inhibition than did the offset condition is contrary to previous reports of equivalent amounts of inhibition (Hoffman \& Stitt, 1980; Stitt, Hoffman, \& Marsh, 1973; Stitt et al., 1974). The differences may lie in the nature of the stimuli employed; for instance, the previous studies used bands of noise, whereas the present study employed pure tones. A study involving a direct comparison of these different stimulus conditions is required before the nature of this difference can be determined.

It seems clear that, in general, this experiment replicated the earlier findings and established some rather interesting and surprising effects. Gaps do have an inhibitory effect, but in the present case no more so than does a simpler component, the offset. A frequency shift across a gap does have a greater inhibitory effect than a gap with no frequency shift, but no more so than a simpler component, the onset.

\section{EXPERIMENT 5}

The previous studies in this sequence made it clear that a gap in a pure tone can inhibit a subsequently elicited blink response. However, if the tone shifts frequency across the gap, even more inhibition is engendered. The purpose of the fifth study was to discern the minimum amount of frequency shift required to produce the additional inhibitory effect above the gap effect. Thus, the amount of frequency shift across the gap was varied and the inhibitory effects were compared with that of two control conditions: a gap with no shift in frequency and the onset of a short tone alone.

\section{Method}

Subjects and Apparatus. Twelve graduate and undergraduate female students from Bryn Mawr College participated in this experiment as paid volunteers. The apparatus was the same as that used in Experiment 1.

Stimulus conditions. During the experimental session, each subject was presented with four basic stimulus conditions, as depicted in Figure 1: (1) tap alone, (2) a 10-sec $1.0-\mathrm{kHz}$ tone with a brief (4-msec) silent period (gap) $154 \mathrm{msec}$ prior to the tap (gap condition), (3) a $1.0-\mathrm{kHz}$ brief $(150-\mathrm{msec})$ tone that offset simultaneously with the onset of the tap (onset condition), and (4) a 10-sec tone in which a brief (4-msec) gap coincided with a downward frequency shift (from one of four frequencies to $1.0 \mathrm{kHz}$ ) $150 \mathrm{msec}$ prior to the tap (shift conditions). The first tone of the shift condition was $2.0 \mathrm{kHz}$ (1 octave higher than the frequency of the $1.0-\mathrm{kHz}$ second tone), $1.25 \mathrm{kHz}$ (1/4 octave higher), $1.12 \mathrm{kHz}$ (1/8 octave higher), or $1.06 \mathrm{kHz}$ (1/16 octave higher). The $2.0-\mathrm{kHz}$ frequency was chosen because it was likely to have a similar effect to the $2.5-\mathrm{kHz}$ tone in the previous studies, and it allowed us to talk easily in term of partial octaves. The smaller octave fractions were employed because Marsh et al. (1975) reported that with bands of noise a 1/4 octave shift significantly inhibited the acoustic startle reflex. There was, then, a total of seven stimulus conditions. The tones had an intensity of $70 \mathrm{~dB}$, and a rise/fall time of $10 \mathrm{msec}$.

Procedure. Each subject received one of each kind of stimulus trial per trial block. Overall, there were seven blocks of seven trials presented in random order that varied from trial block to trial block. Again, colored slides were shown, and the ITI was variable (mean $=20 \mathrm{sec}$; range $=15$ to $25 \mathrm{sec}$ ).

\section{Results and Discussion}

Figure 6 presents the mean response amplitudes averaged across subjects, as a function of amount of frequency shift. It appears that only the largest shift gave equivalent inhibition to the onset, whereas the other shift conditions produced no greater inhibition than did the gap/no-shift condition. A one-way repeated measures analysis of variance confirmed that there were significant differences among the conditions $[F(6,66)=18.5, p<.05]$. Results of the post hoc

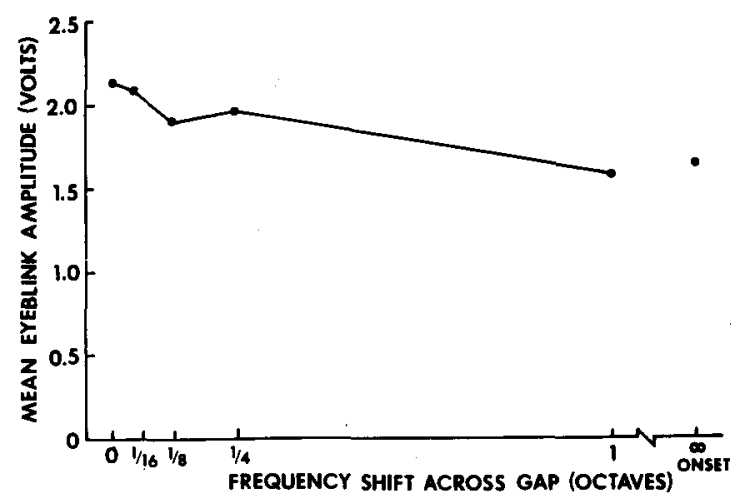

Figure 6. Mesn amplitude of tap-elicited eyeblinks (across subjects) as a function of the amount of frequency shift across a 4 msec gap In acoustic stimulation. 
Newman-Keuls analysis $(\mathrm{p}<.05)$ indicated that the 2.0-1.0-kHz shift and the onset conditions produced significantly more inhibition than any of the other conditions, and that they were equivalent in response amplitude. The other three shift conditions and the gap/no-shift condition resulted in equivalent response amplitudes, and each produced a significant amount of startle inhibition.

The results indicate that only the largest frequency shift (2.0-1.0 kHz) produced the frequency-shift advantage in inhibition over the gap/no-shift condition. The $1 / 4$ octave and smaller shifts did not produce significantly greater inhibition than did the gap/no-shift condition. The threshold for the frequency-shift advantage, then, must lie between 1 octave and a $1 / 4$ octave with respect to the $1.0-\mathrm{kHz}$ tone.

The threshold for the frequency shift advantage in inhibition is much greater than the psychophysically determined just noticable difference (jnd) of between 3 and $7 \mathrm{~Hz}$ for the $1000-$ to $2500-\mathrm{Hz}$ range (Riesz, 1928). Such large jnd values, obtained with the inhibitory paradigm (as opposed to the traditional psychophysical procedures), have also been reported by Marsh et al. (1975) with both frequency and intensity shifts in continuous narrow bands of noise. One possible explanation of the poorer differential thresholds of the inhibitory paradigm relies on the concept of neural sharpening. There is some evidence (Katsuki, 1961) that the average tuning curve (the range of frequencies to which the individual frequency-specific neural units will respond) becomes progressively narrower with more anterior central auditory structures. The proposed mechanism for such neural sharpening is lateral inhibition (von Békésy, 1967). Essentially, the sharpening allows for finer frequency discrimination capacity than might otherwise be expected on the basis of auditory nerve frequency response characteristics. That is, the involvement of higher central auditory structures may be necessary to fine frequency discrimination. This would be consistent with the finding that reflex inhibition is mediated by structures below the meso-diencephalic junction (Leitner, Powers, Stitt, \& Hoffman, 1981), and supports the implications of Experiment 3 that frequency shift detectors are probably not involved in the frequencyshift advantage. That is, with respect to reflex inhibition, the frequency shift advantage appears to be mediated at a relatively low central auditory level, one that is below the levels at which frequency-shift detectors are most frequently found.

\section{REFERENCES}

Búxésy, G. von (1967). Sensory inhibition. Princeton, N.J: Princeton University Press.

Cohen, M. E., Hoffman, H. S., \& StitT, C. L. (1981). Sensory magnitude estimation in the context of reflex modification. Journal of Experimental Psychology: Human Perception and and Performance, 7, 1363-1370.
Davis, M., \& Gendelman, P. M. (1977). Plasticity of acoustic startle response in the acutely decerebrate rat. Journal of Comparative and Physiological Psychology, 91, 549-563.

Dykman, B. M., \& Ison, J. R. (1979). Temporal integration of acoustic stimulation obtained in reflex inhibition in rats and humans. Journal of Comparative \& Physiological Psychology, 93, 939-945.

Groves, P., Boyle, R., Welker, R., \& Miller, S. (1974). On the mechanism of prepulse inhibition. Physiology \& Behavior, $12,367-375$.

Hoffman, H. S., Cohen, M. E., \& StitT, C. L. (1981). Acoustic augmentation and inhibition of the human eyeblink. Journal of Experimental Psychology: Human Perception \& Performance, 7, 1357-1362.

Hoffman, H. S., \& Ison, J. R. (1980). Reflex modification in the domain of startle: I. Some empirical findings and their implications for how the nervous system processes sensory input. Psychological Review, 87, 175-189.

Hoffman, H. S., \& Searle, J. L. (1965). Acoustic variables in the modification of startle reaction in the rat. Journal of Comparative \& Physiological Psychology, 60, 53-58.

Hoffman, H. S., \& STITT, C. L. (1980). Inhibition of the glabella reflex by monaural and binaural stimulation. Journal of Experimental Psychology: Human Perception and Performance, 6, 769-776.

Hoffman, H. S., \& Wible, B. (1969). Temporal parameters in startle facilitation by steady background signals. Journal of the Acoustical Society of America, 45, 7-12.

Ison, J. R. (1982). Temporal acuity in auditory function in the rat: Reflex inhibition by brief gaps in noise. Journal of Comparative and Physiological Psychology, 96, 945-954.

Ison, J. R., \& Pinckney, L. A. (1983). Reflex inhibition in humans: Sensitivity to brief silent periods in white noise. Perception \& Psychophysics, 34, 84-88.

Ison, J. R., Zuckerman, M., \& Russo, J. M. (1975). Combination rules for inhibitory stimuli. Journal of Experimental Psychology: Animal Behavior Processes, 1, 318-325.

KATsuKI, Y. (1961). Neural mechanisms of auditory sensation in cats. In W. A. Rosenblith (Ed.), Sensory communication. New York and Cambridge, Mass: Wiley and M.I.T. Press.

Kelloga, C., Ison, J. R., \& Miller, R. K. (1983). Prenatal diazepam exposure: Effects on auditory temporal resolution in rats. Psychopharmacologia, 79, 332-337.

Kemble, E. D., \& Ison, J. R. (1971). Limbic lesions and the inhibition of startle reactions in the rat by conditions of preliminary stimulation. Physiology \& Behavior, 7, 925-928.

Leitner, D. S., Powers, A. S., Stitt, C. L., \& Hofrman, H. S. (1981). Midbrain reticular formation involvement in the inhibition of acoustic startle. Physiology \& Behavior, 26, 259-268.

LiCRLider, J. C. R. (1951). Basic correlates of the auditory stimulus. In S. S. Stevens (Ed.), Handbook of experimental psychology. New York: Wiley.

Marsh, R. R., Hoffman, H. S., \& StitT, C. L. (1979). Eyeblink elicitation and measurement in the human infant. Behavior Research Methods \& Instrumentation, 11, 498-502.

Marsh, R., Hoffman, H. S., Stitt, C. L., \& Schwartz, G. M. (1975). The role of small changes in the acoustic environment in modifying the startle reflex. Journal of Experimental Psychology: Animal Behavior Processes, 1, 235-244.

Nelbon, P. G., Enulian, S. D., \& Byron, J. S. (1966). Responses of units of the inferior colliculus to time varying acoustic stimuli. Journal of Neurophysiology, 29, 834-860.

Penner, M. J. (1975). Persistence and integration: Two consequences of a sliding integrator. Perception \& Psychophysics, 18, 114-120.

Pernott, D. R., \& Williams, K. N. (1971). Auditory temporal resolution: Gap detection as a function of interpulse frequency disparity. Psychonomic Science, 25, 73-74.

Plomp, P. (1964). Rate of decay of auditory sensation. Journal of the Acoustical Society of America, 36, 277-282.

Riesz, R. R. (1928). Differential intensity sensitivity of the ear for pure tones. Physical Review, 31, 867-875. 
Silverstein, L. D., \& Graham, F. K. (1979). Obicularis oculi excitability and prestimulation effects during REM and NREM sleep. Psychophysiology, 16, 177. (Abstract)

Smiarowski, R. A., \& Carhart, R. (1976). Relations among temporal resolution, forward masking, and simultaneous masking. Journal of the Acoustical Society of America, 60, 423-428.

StitT, C. L., Hoffman, H. S., \& Marsh, R. (1973). Modification of the rat's startle reaction by the termination of antecedent acoustic signals. Journal of Comparative and Physiological Psychology, 84, 207-215.

StitT, C. L., Hoffman, H. S., Marsh, R., \& Boskoff, K. J. (1974). Modification of the rat's startle reaction by an ante- cedent change in the acoustic environment. Journal of Comparative and Physiological Psychology, 86, 826-836.

Whitfield, I. C., \& Evans, E. F. (1965). Responses of auditory cortical neurons to stimuli of changing frequency. Journal of Neurophysiology, 28, 655-672.

Williams, K. N., Elfiner, L. F., \& Howse, W. R. (1979). Auditory temporal resolution: Effects of sensation level. Journal of Auditory Research, 19, 265-269.

(Manuscript received June 10, 1983;

revision accepted for publication October $5,1983$. ) 